\title{
Pulmonale Immunität bei Tuberkulose
}

\section{Pulmonary Immune Mechanisms in Tuberculosis}

Autoren

C. Herzmann ${ }^{1}$, T. Dallenga², U. Kalinke ${ }^{3}$

Institute

1 Forschungszentrum Borstel, Klinisches Studienzentrum, Borstel

2 Forschungszentrum Borstel, Zelluläre Mikrobiologie, Borstel

3 Institut für Experimentelle Infektionsforschung, TWINCORE, Zentrum für Experimentelle und Klinische Infektionsforschung, eine Gemeinschaftseinrichtung von der Medizinischen Hochschule Hannover und dem Helmholtz-Zentrum für Infektionsforschung, Hannover

eingereicht 16.8 .2017

akzeptiert nach Revision 15.11.2017

\section{Bibliografie}

DOI https://doi.org/10.1055/s-0043-122961

Online-Publikation: 21.12.2017 | Pneumologie 2018; 72: 493-502

(c) Georg Thieme Verlag KG Stuttgart · New York ISSN 0934-8387

Korrespondenzadresse

Dr. med. Christian Herzmann, Forschungszentrum Borstel, Parkallee 35, 23845 Borstel

cherzmann@fz-borstel.de

\section{ZUSAMMENFASSUNG}

Tuberkulose wird durch Inhalation von bakterienhaltigen Aerosolen übertragen und 75 \% der Erkrankungen betreffen die Lunge. Die Immunprozesse zwischen Exposition und

Krankheitsentwicklung sind großenteils auf die Lunge beschränkt und nur teilweise entschlüsselt. Der Erreger wird in den meisten Fällen vermutlich durch Zellen des angeborenen Immunsystems kontrolliert oder eradiziert, ohne dass dies mit Standardmethoden in vivo nachgewiesen werden kann. Die anschließende adaptive Immunantwort wird wesentlich von $\mathrm{CD}^{+}$- und $\mathrm{CD} 8^{+}$-T-Lymphozyten vermittelt, während die humorale Immunität nur eine untergeordnete Bedeutung hat. Der Artikel gibt eine Übersicht über den aktuellen Wissensstand der intrapulmonalen Mechanismen, die im Verlauf von Exposition, latenter Infektion, aktiver Erkrankung und Therapie beobachtet werden.

\section{ABSTRACT}

Tuberculosis is transmitted by inhalation of Mycobacterium tuberculosis-containing aerosols; $75 \%$ of all patients show pulmonary manifestation. Immune responses after exposure that lead to clinical symptoms occur mainly in the respiratory tract and are only poorly understood. In most cases, cells of the innate immune system are believed to control the growth of or eradicate inhaled mycobacteria. However, this cannot be verified in vivo using standard methods. Subsequently, $\mathrm{CD} 4^{+}$and $\mathrm{CD} 8^{+} \mathrm{T}$ cell-driven adaptive immune responses are induced that attempt to control bacterial growth. The humoral defence appears to be less important. This article gives an overview of the current understanding of pulmonary immune mechanisms during exposure, latent infection, active disease and therapy of tuberculosis.

\section{Pathogenese}

Über 75\% der Tuberkuloseerkrankungen (TB) betreffen die Lunge. Da diese anatomische Kompartmentalisierung aufwendige Untersuchungsmethoden erfordert, sind bisher nur vergleichsweise wenige Studien durchgeführt worden, sodass das Verständnis der Interaktionen zwischen M. tuberculosis (Mtb) und dem humanen Immunsystem bisher lückenhaft ist. Zudem liegen zwischen pulmonaler Exposition und pulmonaler Erkrankung meist mehrere Jahre, in denen systemische Immunprozesse stattfinden, die für die einzelnen Stadien der Krankheitsentstehung essenziell sind.
Nach einer inhalativen Exposition sind die ersten Schritte der pulmonalen humanen Immunantwort hauptsächlich durch das angeborene Immunsystem charakterisiert. Dabei phagozytieren insbesondere Makrophagen (M $\mathrm{p}$ ) und dendritische Zellen (DZ) in den Alveolen bzw. der Lamina propria des Lungeninterstitiums die inhalierten Bakterien. Auch in späteren Stadien der Erkrankung bleiben $M \varphi$ und DZ als zelluläre Nische für Mtb erhalten, während dann zusätzlich auch anderen Zellen der angeborenen Immunität eine Bedeutung zukommt, insbesondere den T-Lymphozyten, die im regionalen Lymphknoten den Priming-Prozess durchlaufen. Die latente Infektion, die Bildung von Granulomen bis hin zur aktiven Erkrankung wird anschlie- 
ßend maßgeblich durch $\mathrm{CD}^{+}$- und $\mathrm{CD} 8^{+}$-T-Lymphozyten der adaptiven Immunität geprägt. Daneben sind Mukosa-assoziierte invariante T-Zellen (MAIT), regulatorische T-Zellen ( $\left.T_{\text {regs }}\right)$ und unkonventionelle T-Lymphozyten in die Pathogenese involviert. Obwohl viele dieser Zellen polyfunktional sind, kommt bei der antimykobakteriellen Wirksamkeit den proinflammatorischen Typ-1-Zytokinen, insbesondere dem Interferon-y (IFN-y), eine besondere Bedeutung zu [1]. Es ist weiterhin bekannt, dass auch das angeborene Immunsystem essenziell für die Pathogenese ist. Natürliche Killerzellen fördern die antimykobakterielle Kapazität der $M \varphi$ und kontrollieren Mtb über verschiedene zytotoxische Mechanismen [2]. Neutrophile finden sich in großer Zahl in der bronchoalveolären Lavage (BAL) und im Sputum von Tuberkulosepatienten [2]. Die Bedeutung einzelner Aspekte der Immunantwort für den Krankheitsverlauf von TB wird durch immunsupprimierte Patienten verdeutlicht, bei denen entweder eine Infektion mit HIV, eine immunmodulierende Therapie oder ein angeborener Defekt vorliegt und bei denen die TB einen besonders schweren Verlauf nimmt.

Diese klinischen Beobachtungen zeigen auf, dass die antimykobakteriellen Prozesse in der Lunge vielschichtig sind, zumal Mtb zahlreiche Strategien entwickelt hat, einzelne Abwehrmechanismen zu umgehen oder außer Kraft zu setzen. Hierzu gehören die Blockade der Reifung des Phagosoms, um so intrazelluläres bakterielles Wachstum zu ermöglichen, die Hemmung oder Aktivierung der Apoptose, die Störung des Inflammasoms und andere [2]. Dieser Artikel fasst den gegenwärtigen Kenntnisstand pulmonaler Immunmechanismen nach Kontakt mit Mtb zusammen.

\section{Inhalation}

Die Exposition gegenüber Aerosolen, die Mtb enthalten, kann vermutlich nur dann zur Infektion führen, wenn die PartikelgröBe der Aerosole eine Inhalation der Bakterien bis in die Alveolen ermöglicht ( $1-5 \mu \mathrm{m})$, also bspw. beim Hustenstoß [3]. Interessanterweise ergeben sich aus Tierexperimenten Hinweise, dass größere, mit Mykobakterien belastete Partikel zu einer Immunisierung über die Mundschleimhaut und der Induktion einer protektiven pulmonalen Immunantwort führen können [4].

\section{Phagozytose und Eradikation}

$M t b$ wird in den Alveolen unter anderem von antigenpräsentierenden Zellen (APZ) - vorrangig $M \varphi$, aber auch verschiedene Typen von DZ - phagozytiert ( $\mathbf{A} \mathbf{b} \mathbf{b}$. 1). Alle Zellen der angeborenen Immunität, aber auch einige Zellen der adaptiven Immunität (z.B. Lymphozyten) verfügen über Pattern Recognition Rezeptoren (PRR), die es ermöglichen, definierte Strukturmerkmale des Erregers (Pathogen-Associated Molecular Patterns, PAMP) zu erkennen. Hierzu gehören Toll-Like Receptors (TLR), intrazelluläre Nucleotide-binding Oligomerization Domain (NOD)-Like Receptors (NLR), Retinoic Acid Inducible Gene ILike Receptors (RLR) und C-Typ Lektin Rezeptoren (CTLR). Die Aktivierung der PRR auf der Zelloberfläche oder im Zytosol triggert zelluläre Abwehrmechanismen (Phagozytose, Autophagie, Apoptose, Cathelicidinproduktion, Aktivierung von NF-kB und
Inflammasoms, etc.) [5] und bahnt so den weiteren Verlauf der Immunantwort an. So sind TLR-2, TLR-6, TLR-8, TLR-9, NLR und CTLR durch Bindung von Lipoproteinen an der Erregereradikation beteiligt [6]. Die Signalkaskade führt über MyD88 und NF-kB zur Sekretion proinflammatorischer Zytokine wie TNF- $\alpha$, IL-1 $\beta$ und Typ-1-Interferonen [5]. Zu den CTLR gehört auch der Mannoserezeptor, der mannosyliertes Lipoarabinomannan, Phosphatidyl-Inositol-Mannoside und Lipomannan bindet und möglicherweise primär für die Aufnahme nicht-opsonierter Mykobakterien verantwortlich ist [5]. Im günstigsten Fall erfolgt nun eine frühe Eradikation („early clearance“) der Erreger. Genetische Einflüsse auf Seiten des Wirts werden für die Induktion einer frühen effektiven Immunantwort diskutiert [7]. Einer derzeitigen Hypothese zufolge wird Mtb von mehr als der Hälfte der exponierten Personen durch das angeborene Immunsystem aus den Atemwegen eradiziert [8]. Ein weiterer Teil exponierter Personen entwickelt eine regionale adaptive Immunantwort, die jedoch nicht systemisch messbar ist, was mit dem Begriff „incipient tuberculosis“ beschrieben wurde [9]. Kernstück der angeborenen zellulären Immunität ist die Abtötung der Erreger im Phagosom, also in zellulären Organellen, in denen der enzymatische Abbau phagozytierten Materials stattfindet. Der hierfür erforderliche Reifungsprozess der Phagosomen erfordert die Fusion mit Lysosomen, die Ansäuerung des Phagosoms durch Protonenpumpen und die Aktivierung lysosomaler Hydrolasen [10]. Von Bedeutung sind hierfür die $\mathrm{H}^{+}$-ATPase in den Vakuolenmembranen und Cathepsin D, deren Hemmung zu einem verstärkten intrazellulären Bakterienwachstum führt [10]. Mtb kann diesen Prozess blockieren und sich so einem Angriff durch die angeborene Immunität entziehen. Virulente Bakterien können zudem durch Störung der Zellmembranreparatur die Nekrose infizierter $M \varphi$ auslösen, was zu einer weiteren Verbreitung der Erreger im Körper führen kann, während ein geordneter apoptotischer Zelltod infizierter Zellen zur Abtötung der Erreger führt. Phagozyten verfügen über ein breites Arsenal antibakterieller Strategien. Der Wirtsfaktor Hepcidin weist innerhalb der Zelle bspw. eine räumliche Nähe zu Phagosomen auf und wirkt in durch IFN-y aktivierten $M \varphi$ antimykobakteriell [11]. Andere Mechanismen umfassen die Aktivierung von Nikotinadeninphosphatoxidase und StickstoffmonoxidSynthetase, die Stickstoff- und Sauerstoffradikale erzeugen. Neben den proinflammatorischen Zytokinen IFN- $\gamma$ und TNF- $\alpha$ scheint hierfür auch 1,25-Hydroxy-Vitamin D3 erforderlich zu sein [5]. Zudem wird dem durch Vitamin D3 induzierbaren Cathelicidin LL37 eine antimykobakterielle Wirkung zugeschrieben, die von IFN- $\gamma$ und TNF- $\alpha$ unabhängig ist [12]. IFN- $\gamma$ und das nachgeschaltete LRG-47 führen ferner zur Induktion von Autophagie, die durch Fusion von Phagosomen und Lysosomen das bakterielle Wachstum hemmen kann [13].

Neben den Wirtsfaktoren beeinflussen auch bakterielle Eigenschaften wie die Infektionsdosis und die mykobakterielle Membranstruktur den Verlauf der Immunantwort. Bei höheren Infektionsdosen mit Mtb oder M. bovis Bacillus Calmette-Guérin (BCG) wurde in humanen und murinen $M \varphi$ eine schlechtere Wachstumskontrolle beobachtet als bei kleineren Dosen, was teils durch eine verminderte Azidifizierung des Phagosoms erklärbar ist $[10,14]$. 
Da das zu den CTLR gehörende dendritic cell-specific intercellular adhesion molecule-3-grabbing nonintegrin (DC-SIGN) mit unterschiedlicher Affinität an zelluläre Bestandteile unterschiedlicher Mykobakterienspezies bindet, können auch strukturelle Unterschiede der Bakterien den Verlauf der Infektion beeinflussen [5, 15]. Das Bindungsverhalten von mannosyliertem Phosphatidyl-Myo-Inositol Mannosid, Lipomannan und mannosyliertem Lipoarabinomannan ist auch von der Struktur der Lipoglykoside, der terminalen Carboxilierung und dem Grad ihrer Acylisierung abhängig [16].

\section{Induktion der adaptiven Immunität}

Am Übergang der angeborenen zur adaptiven Immunität, die im Tiermodell erst ca. zwei Wochen nach Inhalation und Phagozytose beobachtet wird, präsentieren infizierte $M \varphi$ und DZ im drainierenden Lymphknoten mykobakterielle Antigene auf Major Histocompatibility Complex (MHC) I- und II-Molekülen [17]. Es ist bisher unklar, welche Peptidepitope dort während des Primings präsentiert werden müssen, um eine protektive T-Zellantwort zu erzeugen. Schon in latent infizierten, nicht erkrankten Kontaktpersonen wurden 82 Antigene identifiziert, gegen die sich die T-Zellantwort bei 75\% der Personen richtet [18].

Wie lange Mtb-spezifische Lymphozyten in der Lunge verbleiben und dort eine regionale, nicht jedoch systemische Immunantwort zeigen (sog. incipient TB), ist bisher unklar [9]. Nach dem Priming folgt eine Phase klonaler Expansion, während der die T-Lymphozyten den Lymphknoten verlassen, im Blut zirkulieren und so das pulmonale Gefäßbett erreichen [17]. Dort migrieren sie in die Lunge und übernehmen eine zentrale Rolle bei der Granulombildung bzw. der Aktivierung und Orchestrierung der phagozytierenden Zellen. Ob das adaptive Immunsystem - pulmonal oder systemisch - in der Lage ist, Mykobakterien zu eradizieren oder nur innerhalb von Granulomen zu kontrollieren, ist bislang unbekannt [19]. Auf Zytokinebene sind IFN-y und IL-12 bei der Kontrolle der Infektion durch CD4 ${ }^{+}$-T-Zellen essenziell, wobei IFN- $\gamma$ eine besonders wichtige Rolle zukommt.

\section{Granulombildung}

Histologisch sind verkäsende Granulome das herausragende Kennzeichen einer Mtb-Infektion. Sie bestehen aus klassischen Makrophagen und Makrophagen, die zu Epitheloidzellen ausdifferenziert sind sowie aus mehrkernigen Riesenzellen (Langerhans Zellen) und sind umgeben von einem Ring von Lymphozyten. In einem blutzellbasierten In-vitro-Modell zeigten latent infizierte Individuen eine deutlich stärkere Granulombildung, eine bessere bakterielle Wachstumskontrolle und höhere IFN-y- und IL-12-Konzentrationen als nicht infizierte Menschen [20]. Allerdings ist aus histologischen Studien an Makaken und PET-CT-Untersuchungen an Patienten bekannt, dass immer Granulome verschiedener Entwicklungsstadien parallel existieren, die auch im Verlauf wechselnde Aktivitäten zeigen [21 23]. Initial werden nicht infizierte Makrophagen über die Metalloproteinase (MMP) 9 rekrutiert, die von Epithelzellen in unmittelbarer Umgebung von infizierten Makrophagen sezerniert wird [24]. Dies führt jedoch nicht zu einer Erregereindämmung, sondern zu weiterer bakterieller Vermehrung und zu einer vermehrten Infektion von Makrophagen. Diese Phase der frühen Dissemination dauert im Makakenmodell ungefähr 6 Wochen [22]. Makrophagen müssen einen epithelialen Transformationsprozess durchlaufen, um eine geordnete Granulomstruktur bilden zu können [25]. In späteren Infektionsstadien kann die Mehrzahl der Granulome die Bakterien zwar eindämmen, jedoch wurde ein größerer Granulomdurchmesser sechs Wochen nach Infektion als Risikofaktor für die Entwicklung einer aktiven Tuberkulose identifiziert [22]. Im Makakenmodell zeigten im Granulom nur $8 \%$ der Lymphozyten eine Mtb-Spezifität, jedoch fanden sich viele polyfunktionale T-Zellen, deren Zytokinmuster (vermehrte Ausschüttung von IL-17, TNF- $\alpha$, INF- $\gamma$, IL-2) mit mikrobiologischer Sterilität assoziiert war. Die Bedeutung von TNF- $\alpha$ für die Instandhaltung der Granulome ist aus der Therapie mit anti-TNF- $\alpha$-Wirkstoffen bekannt, die das Erkrankungsrisiko innerhalb weniger Wochen bis um das 25-Fache steigern können. Interessanterweise waren insbesondere T-Zellen, die proinflammatorische (IL-17) und antiinflammatorische (IL-10) Zytokine ausschütteten, mit bakterieller Eradikation assoziiert. Diese Aussage wird von Mausexperimenten gestützt, in denen die Blockade von IL-10 in den frühen Infektionsstadien zu insuffizienter Granulombildung und schwereren Krankheitsverläufen führte [26]. Nur ein Teil der Granulome führt in späteren Infektionsstadien zur Antigenpräsentation im regionalen Lymphknoten und somit zur Induktion der adaptiven Immunität, durch die die latente Infektion definiert ist.

\section{Latente Infektion}

In der klinischen Praxis wird die latente Mtb-Infektion durch einen Interferon-y Release Assay (IGRA) definiert. Bis vor einigen Jahren war auch der weniger spezifische Tuberkulinhauttest (THT) gängige Praxis. Das Ergebnis des THT ist durch den Durchmesser der Induration definiert, die an der Injektionsstelle nach 72 Stunden durch zelluläre Infiltration als Reaktion auf eine intrakutane PPD (Purified Protein Derivate)-Injektion entsteht. Der IGRA misst das Schlüsselzytokin, nämlich die Interferon-y (IFN- $\gamma$ )-Ausschüttung aus T-Lymphozyten aus dem Blut, die in vitro auf Mtb-spezifische Peptidantigene reagieren (u.a. ESAT-6 und CFP-10). Sofern die exponierte Person keine klinischen, radiologischen oder mikrobiologischen Befunde einer TB aufweist, wird hierüber die latente Tuberkuloseinfektion (LTBI) definiert.

Trotz der herausragenden Bedeutung des IFN- $y$ ist die Relevanz eines positiven IGRA-Befundes begrenzt, da der Vorhersagewert für die Entwicklung einer aktiven TB nach Exposition gering ist [27]. Zudem fällt bei Patienten mit aktiver TB der Blut-IGRA häufig negativ aus [28]. Der Elispot-basierte IGRA TSpot.TB ${ }^{\circledR}$ weist jedoch auf eine aktive Erkrankung hin, wenn sich in der bronchoalveolären Lavage (BAL) im Vergleich zum Blut eine Anreicherung TB-spezifischer Lymphozyten findet [29]. 


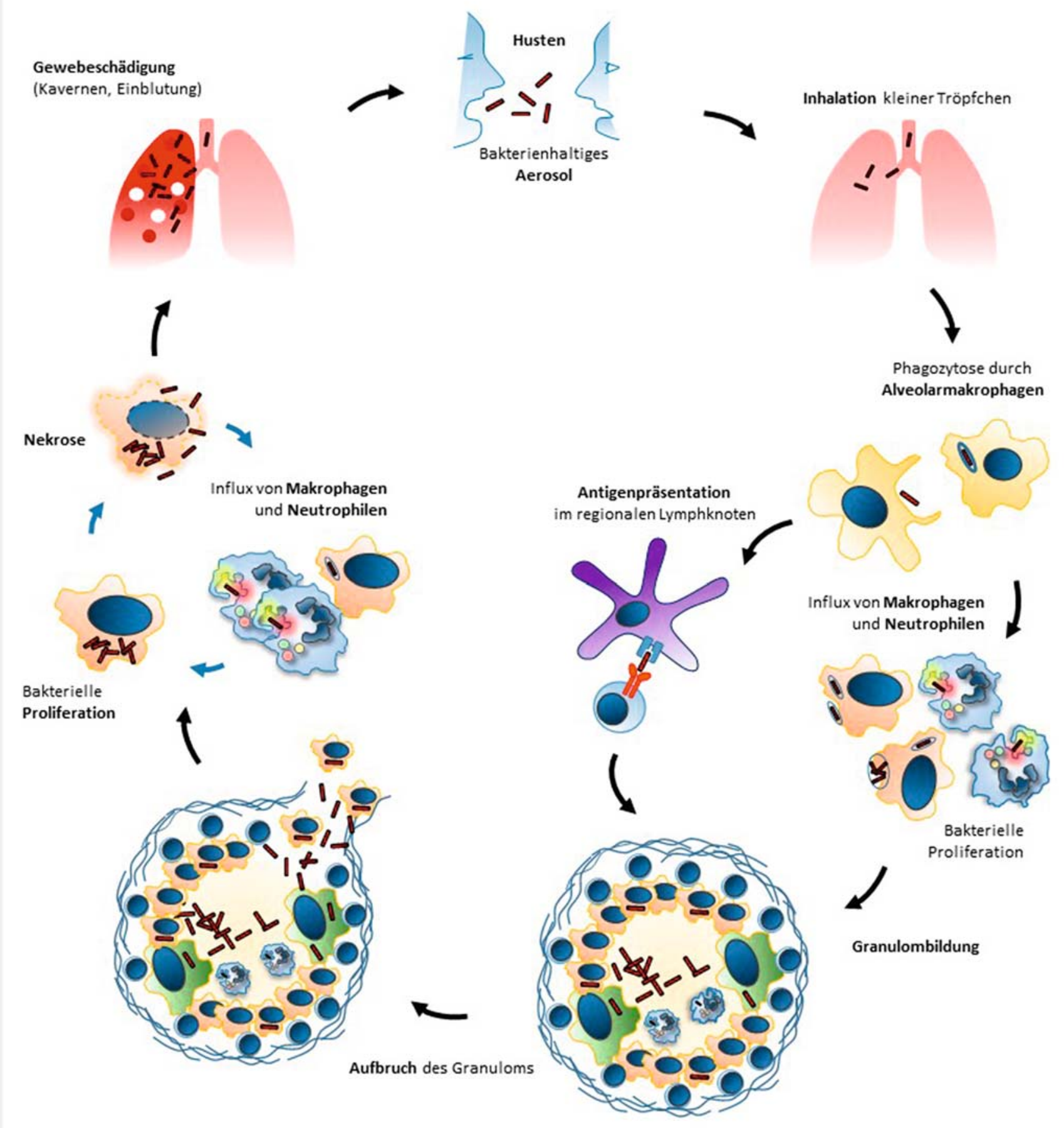

- Abb. 1 Infektionskreislauf von M. tuberculosis (rot). Inhalation eines bakterienhaltigen Aerosols, erzeugt meist durch hustende Patienten. Partikel bis $5 \mu \mathrm{m}$ gelangen in die Alveolen, wo sie von Alveolarmakrophagen (gelb) und dendritischen Zellen (violett) phagozytiert werden. Nach kurzer Wachstumsphase im Phagosom der Phagozyten werden v. a. Monozyten/Makrophagen (orange) und Neutrophile (blau) rekrutiert. Im regionalen Lymphknoten erfolgt das Priming der T-Lymphozyten. In hypoxischen Granulomen wird die Infektion zunächst räumlich und zeitlich kontrolliert. Diese bestehen aus einem nekrotischen Zentrum mit zahlreichen extrazellulären Mykobakterien, umgeben von infizierten Makrophagen und Schaumzellen (grün), die wiederum umgeben sind von T-Zellen (dunkelblau). Umschlossen ist das Granulom von fibrösem Kollagen („fibrous cuff“). Trigger (z. B. Immunsuppression) können zum Aufbruch des Granuloms mit Freisetzung von M. tuberculosis führen, wodurch es zu massivem Influx von größtenteils Monozyten/Makrophagen und Neutrophilen kommt. Es folgt ein Kreislauf von intrazellulärer bakterieller Proliferation gefolgt vom nekrotischen Zelltod. Die Freisetzung gewebsschädigender Moleküle aus nekrotischen Neutrophilen verstärkt die Pathologie: Ausbildung von Kavernen (weiße Kreise), Auftreten von pulmonalen Hämorrhagien (rote Kreise) und Aerosolbildung beim Husten. 


\section{Interferon- $\gamma$}

Die zentrale Rolle von IFN-ץ zeigt sich an Individuen mit primären Defekten in der IFN-y und IL-12 Signalkaskade, die eine erhöhte Sensitivität für mykobakterielle Infektionen haben [30]. Patienten mit angeborenen Immundefekten, die die IL-12/23IFN- $\gamma$-Integrität von $\mathrm{M} \varphi$ und dadurch deren Zusammenspiel mit T- und NK-Zellen beeinträchtigen, neigen zu schweren mykobakteriellen Infektionen durch Erreger, die für gewöhnlich als wenig virulent eingestuft werden (nicht tuberkulöse Mykobakterien, M. bovis BCG) [31]. Entsprechend können auch Mäuse mit Defekten in den Genen von IFN-y und IL-12 eine Mtb-Infektion nicht kontrollieren. In adoptiven Transferexperimenten mit Mäusen konnte gezeigt werden, dass das von CD4 ${ }^{+}-T-Z e l l e n$ produzierte IFN-y für die Kontrolle von Mtb entscheidend ist [32]. Allerdings zeigen Studien, dass die Menge an produziertem IFN-y kein Korrelat für Protektion darstellt: In einer klinischen Studie in Kapstadt mit mehr als 5000 Kindern hatte die nach $M$. bovis BCG-Impfung gebildete Menge an IFN-y keine Aussagekraft über die Wahrscheinlichkeit, während der ersten zwei Lebensjahre nicht an TB zu erkranken [33]. Zudem entwickelten Personen mit sehr hoher IFN-y-Antwort vermehrt eine TB [34]. In einer Impfstudie mit viralen Vektoren, die ein Mtb-Antigen exprimierten, wurden spezifische T-Zellen induziert, die große Mengen IFN-y exprimierten; dennoch induzierte der Impfstoff keinen Schutz [35]. Diese Befunde belegen, dass IFN-y notwendig ist, um Mtb zu kontrollieren, die Stärke der IFN-y-Antwort aber nicht mit der Resistenz gegen eine TB korreliert.

\section{Regulatorische T-Zellen}

Regulatorischen T-Lymphozyten ( $\mathrm{T}_{\text {regs }}$ ) wird eine modulierende Rolle in der adaptiven Immunität zugeschrieben, deren Ziele die Feinabstimmung der zellulären Aktivitäten, die Verhinderung von Gewebeschädigung und die Beendigung einer erfolgreichen Immunantwort sind. Sie sind antigenspezifisch, jedoch sind zahlreiche Zellpopulationen von ihrer immunmodulatorischen Funktion betroffen, darunter Th1-, Th2-, Th17- und үסTZellen, deren Aktivität oder Proliferation eingeschränkt wird.

Bereits bei Kontaktpersonen mit LTBI finden sich signifikant mehr CD4 + CD25 + CD127 - $\mathrm{T}_{\text {regs }}$ in der BAL als bei exponierten Probanden mit negativem IGRA-Befund [36]. In Ex-vivo-Infektionsexperimenten mit alveolären $M \varphi$ und $M t b$ führte die $\mathrm{Zu}$ gabe von $\mathrm{T}_{\text {regs }}$ aus dem Blut des gleichen Probanden zu vermehrten mykobakteriellen Wachstumsraten [37]. Dies ließ sich auch in vivo im Mausmodell für pulmonale $T_{\text {regs }}$ nachvollziehen, was den Schluss nahelegt, dass pulmonale $T_{\text {regs }}$ funktional aktiv sind und nicht nur „in Rufbereitschaft“ (Bystander) in die Lunge rekrutiert werden, um gewebezerstörende Immunreaktionen einzudämmen [38]. Nach derzeitigem Verständnis schwächen funktional aktive $T_{\text {regs }}$ somit die Immunantwort in der Lunge, was zu vermehrtem bakteriellen Wachstum führt und ggf. die Entstehung einer aktiven TB begünstigt. Allerdings

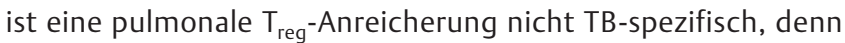
auch bei Sarkoidose und Lungenkarzinom finden sich vermehrt pulmonale $T_{\text {regs }}$.

\section{Aktive Tuberkulose}

Die Entstehung einer aktiven Tuberkulose ist von vielfältigen Faktoren abhängig, die im Folgenden dargestellt werden und die Beteiligung zahlreicher immunologisch aktiver Zellpopulationen illustrieren.

\section{Lymphozyten}

Nicht nur bei der latenten, sondern ebenfalls bei der aktiven Erkrankung kommt den T-Lymphozyten eine zentrale Rolle zu. Die Rolle des lymphozytären IFN- $\gamma$ wurde bereits oben dargestellt. CD4 ${ }^{+}$-T-Zellen benötigen direkten Kontakt mit infizierten APZ, um Mtb unter Kontrolle zu bringen. Dies belegt ein Mausmodell, in dem gemischte Knochenmarkschimären, die sowohl MHC-II-positive als auch MHC-II-negative M $\varphi$ und DZ enthalten, mit Mtb infiziert wurden [39]. Dabei wurde festgestellt, dass in den Lungen infizierter Tiere MHC-II-negative Zellen eine höhere Erregerlast zeigten als MHC-II-positive, und dass nach Depletion von $\mathrm{CD}^{+}$-T-Zellen beide Zelltypen gleich stark infiziert waren. Auch von HIV-infizierten Patienten wissen wir, dass nicht die abnehmende Zahl, sondern die eingeschränkte Funktion zirkulierender CD4+-Zellen das Risiko einer TB erhöht. In der BAL nehmen polyfunktionale IFN- $\gamma$, TNF- $\alpha$ und IL-2 exprimierende Zellen $a b$, allerdings hat dies nach gegenwärtigem Kenntnisstand keinen Einfluss auf die Wachstumsraten von Mtb in alveolären $\mathrm{M \varphi}$ [40]. Die Abnahme polyfunktionaler T-Zellen wurde von Jambo et al. bestätigt, was auf eine eingeschränkte regionale Immunantwort schließen lässt [41]. Für diese regionale Immunität ist die Eigenschaft von CD4 ${ }^{+}$-T-Zellen, die Zirkulation zu verlassen, um in das Lungengewebe einzuwandern, wahrscheinlich von größter Bedeutung. Mittels Fluorochrommarkierter Antikörper, die einem Tier wenige Minuten vor der Entnahme der Lunge intravenös verabreicht werden, können in Lysaten präparierter Organe intravasale Zellen markiert werden. Hierüber konnten die relevanten $C D 4^{+}$-T-Zellen der Lunge weiter charakterisiert werden [42]. In der Maus exprimieren intravasale T-Zellen in der Lunge viel KLRG1 und sind somit enddifferenziert. Weiterhin exprimieren sie den Chemokinrezeptor CX3XR1 und können sehr viel IFN-y produzieren. Dagegen exprimieren extravasale T-Zellen im Lungenparenchym den Chemokinrezeptor CXCR3 und zeigen eine besonders hohe Expression von Aktivierungsmarkern wie CD69 und PD-1.

Nach adoptivem Transfer von intravasalen CX $3 C R 1^{+} \mathrm{CD} 4^{+}-\mathrm{T}$ Zellen fanden sich nur wenige dieser Zellen im Lungenparenchym wieder, während adoptiv transferierte parenchymale CXCR3 ${ }^{+}$-T-Zellen zurück ins Lungenparenchym wanderten. Obwohl sie geringere Mengen an IFN-y produzierten, vermittelten die parenchymalen CD4 ${ }^{+}-\mathrm{T}$-Zellen einen besseren Schutz vor einer Mtb-Infektion als die vaskulären $C D 4^{+}-T-Z e l l e n$. Allerdings wurde schon in früheren Arbeiten beschrieben, dass PD-1-defiziente Mäuse, in denen die IFN-y-Produktion von T-Zellen durch PD-1-Bindung nicht reduziert wird, schwerere $M t b$-Infektionen als Wildtyptiere zeigen. Das lässt den Schluss zu, dass parenchymale CXCR3 ${ }^{+} \mathrm{CD} 4^{+}$-T-Zellen nicht nur Schutz vor Mtb vermitteln, sondern auch Schaden anrichten können. Neue Arbeiten bewiesen, dass die IFN-y-Produktion parenchymaler CXCR3 ${ }^{+}$ CD4 ${ }^{+}$-T-Zellen über $\mathrm{PD}-1$ reduziert werden muss, um eine leta- 
le TB zu vermeiden [43]. Allerdings inhibieren diese Zellen in der Maus Mtb weniger in der Lunge als vielmehr in der Milz. Somit bleibt weiterhin ungeklärt, wie das Zusammenspiel von vas-

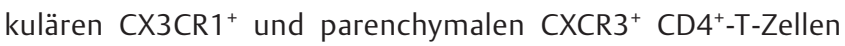
funktioniert.

Neben CD4+-T-Zellen können in Tiermodellen auch CD8 ${ }^{+}$-TZellen wichtige Funktionen bei der Immunabwehr von Mtb übernehmen. Frühe adoptive T-Zell-Transferexperimente ließen vermuten, dass CD8 ${ }^{+}$-T-Zellen Schutz vor Mtb-Infektionen vermitteln können. Im Nagermodell ( $\beta 2-\mathrm{m}^{-/-}$Mausmutanten) wurde schließlich eine protektive Wirkung nachgewiesen [44]. CD8 ${ }^{+}$-T-Zellen können nicht nur zytotoxische Moleküle (z.B. Perforin), sondern auch vielfältige Zytokine exprimieren und so das Milieu infizierter Gewebe verändern. In einer brasilianischen Studie mit 36 Patienten korrelierte die Zytokinexpression von $\mathrm{CD}^{+}$-T-Zellen mit der Bakterienlast: Patienten mit hoher Erregerlast zeigten CD8 ${ }^{+}$-T-Zellen mit starker IL-10- und TGF- $\beta$-Expression. Zudem zeigte die Studie, dass CD8 ${ }^{+}-\mathrm{T}$-Zellen bei TB-Patienten IFN-y und IL-17 produzieren [45]. In einem persistenten Infektionsmodell konnte zudem gezeigt werden, dass besonders CD8 ${ }^{+}$-T-Zellen die Reaktivierung von Mtb-Erregern unterdrücken. Im Mausmodell führte die Depletion von CD8 ${ }^{+}$-T-Zellen während der persistenten Phase der Infektion, nicht jedoch die Depletion von CD4 ${ }^{+}$-T-Zellen zu einer erhöhten Bakterienlast in der Lunge [46].

Auch die Häufigkeit der $T_{\text {regs }}$ im Blut verändert sich bei aktiver Tuberkulose, jedoch berichten einige Studien von einer Abnahme, andere von einer Zunahme der $\mathrm{T}_{\text {regs }}[47,48]$. Auch in der Lunge fand sich bei pulmonaler TB eine Anreicherung von $\mathrm{T}_{\text {regs }}$ [37]. Eine chinesische Studie an Han-Chinesen legt nahe, dass Mutationen des von $\mathrm{T}_{\text {regs }}$ exprimierten CTLA-4 das TB-Risiko beeinflussen [49]. Therapeutische Ansätze einer CTLA-4-Antagonisierung, wie sie in der Onkologie üblich sind, werden derzeit in der TB-Therapie nicht verfolgt. Das hängt damit zusammen, dass in einer eine älteren Mausstudie die mykobakterielle Eradikation aus der Lunge durch CTLA-4-Antogonisierung nicht verbessert wurde [50].

Trotz großer Fortschritte bei der Untersuchung der Rolle von T-Zellen und der Charakterisierung von lungenständigen T-Zellen bleibt es unklar, welche Faktoren im Menschen den Schutz vor einer Infektion mit Mtb vermitteln. Weitere Studien sind notwendig, um diese Frage zu beantworten.

\section{Neutrophile Granulozyten}

Neutrophile Granulozyten stellen mit 60 - 70\% den größten Anteil der Leukozyten im Blut dar. Sie wandern entlang eines CXCL8-Gradienten, der über die Rezeptoren CXCR1 und CXCR2 erkannt wird, in den Infektionsherd ein und phagozytieren eingedrungene Bakterien, die sie mittels ihres antimikrobiellen Arsenals (reaktive Sauerstoffspezies, antimikrobielle Peptide, Hydrolasen, Proteinasen, Lipasen, Phosphatasen, Kollagenasen und Matrix-Metalloproteasen) rasch abtöten [51]. Neutrophile spielen v.a. während der frühen Phase einer Reaktivierung der TB eine Rolle, die sich in einer postprimären Pneumonie äußert. Nach Reaktivierung, z.B. durch Aufbrechen eines Granuloms oder einer Kaverne, kommt es zu einem massiven Influx von Neutrophilen. Neutrophile sind die am häufigsten vorkommen- den Zellen im Sputum und der BAL von Patienten mit aktiver Lungen-TB, und es sind die Neutrophilen, die die größte Bakterienlast tragen [52]. Histologische Schnitte von tuberkulösen Lungen zeigten v.a. S100A8/9+-Neutrophile in inflammatorischen Granulomen, deren Anzahl mit der Entzündung korrelierte [53]. Die Serumlevel eines weiteren Alarmins, S100A12, konnte als unabhängiger Prädiktor nicht nur für die Schwere des Krankheitsverlaufs und das Therapieansprechen genutzt werden, sondern korrelierte auch mit radiologischen Infiltraten [54]. Berry et al. identifizierten ein TB-spezifisches mRNA-Profil des Blutes von Patienten, das mit dem radiologischen Ausmaß der Erkrankung korrelierte und sich unter erfolgreicher Behandlung normalisierte [55]. Dieses Profil war dominiert von einer Neutrophilen-assoziierten Signatur. Unterstützend fanden Gopal et al., dass S100A8/A9-produzierende Neutrophile dominant in Granulomen von TB-Patienten vorkommen und dass S100A8/A9-Serumspiegel mit CXCL8-Serumspiegeln, der Neutrophilenzahl im Blut und der Lungenschädigung korrelierten [53].

Obwohl zahlreiche Neutrophile in den Infektionsherden vorhanden sind, können sie Mtb nicht kontrollieren. Neuere Studien weisen eher darauf hin, dass deren toxisches Arsenal zur Gewebeschädigung beiträgt, was zu einem aggressiveren Krankheitsverlauf, Gewebeschädigung und schließlich zur Infektion weiterer Individuen führt [56]. Unsere Gruppe hat beobachtet, dass humane Neutrophile, nachdem sie in vitro mit einem virulenten Stamm von Mtb infiziert wurden, nicht in der Lage sind, die Bakterienlast zu reduzieren [57]. Stattdessen sterben sie einen schnellen nekrotischen Zelltod, der von reaktiven Sauerstoffspezies abhängig ist. Wir gehen davon aus, dass Mtb durch die rasche Induktion von Nekrose dem hochwirksamen bakteriziden Arsenal der Neutrophilen entkommt [58]. Reaktive Sauerstoffspezies stellen einen der wichtigsten Abwehrmechanismen der Neutrophilen dar. Durch die Sekretion des Enzyms Catalase, das rasch Wasserstoffperoxid in Wasser und Sauerstoff zerlegt, ist Mtb vor reaktiven Sauerstoffspezies geschützt [59].

\section{NK-Zellen, MAIT-Zellen und $y \delta-T-Z e l l e n$}

Natürliche Killerzellen (NK-Zellen) sind zytotoxische Lymphozyten des angeborenen Immunsystems, die infizierte Zellen töten können. Anders als T-Zellen besitzen sie keinen antigenspezifischen Rezeptor und müssen nicht antigenabhängig geprimt werden. NK-Zellen wurden in Lungengranulomen in histologischen Schnitten von Patienten mit pulmonaler TB nachgewiesen. Humane NK-Zellen, die ex vivo mit Glutathion aktiviert wurden, waren in der Lage, das Wachstum von Mtb in Monozyten zu kontrollieren [60]. Dies war abhängig von der Produktion der zytotoxischen Liganden FasL und CD40L [61], aber auch von sezernierten Molekülen wie Perforin, Granulysin und Granzyme [62]. Eine andere Studie zeigte allerdings, dass Monozyten von TB-Patienten die lytische Aktivität und IFN-y-Produktion von NK-Zellen herunterregulieren [63]. Dies wurde im Tiermodell untermauert, in dem deren Depletion keinen Einfluss auf die Erregerlast in der Lunge hatte [64]. 
Ähnlich den NK-Zellen besitzen die mukosal-assoziierten invarianten T-Zellen (MAIT-Zellen) keine antigenspezifischen Rezeptoren, stattdessen exprimieren sie konservierte irrelevante Antigen-Rezeptoren. Die IFN-y-Produktion von MAIT-Zellen von TB-Patienten ist gegenüber gesunden Kontrollen nach Stimulation mit mykobakteriellen Lysaten reduziert [65]. Im Tiermodell konnte eine Anreicherung von MAIT-Zellen in den Lungen infizierter Mäuse gezeigt werden, die dort zur frühen Protektion gegen TB beitrugen [66].

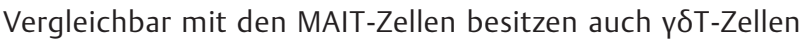
einen semi-invarianten T-Zellrezeptor, der allerdings im Gegensatz zu klassischen T-Zellen aus einer $\gamma$ - und einer $\delta$-Kette besteht. үठT-Zellen reagieren spezifisch auf mykobakterielle Antigene und spielen eine wichtige Rolle bei der Protektion. In entstehenden Granulomen sezernieren sie Immunmodulatoren und vernetzen die angeborene mit der adaptiven Immunität, unter anderem durch Aktivierung von $M \varphi$ und DZ, die wiederum Mtb-spezifische CD4+-T-Zellen aktivieren [67]. Bei Patienten mit aktiver TB sind $ү \delta T$-Zellen unreif und ihre Anzahl ist erniedrigt [68].

\section{B-Lymphozyten}

Die Infektion mit Mtb geht mit der Bildung von Antikörpern einher; die Bedeutung der humoralen Immunität im Menschen ist jedoch unklar [69]. Dies beruht unter anderem auf dem historisch gewachsenen Paradigma, dass die zelluläre Immunität den wesentlichen Beitrag zur Elimination des Erregers leistet und auf der Tatsache, dass im wissenschaftlichen Konsens eine LTBI durch Parameter der zellulären Immunität (positiver Tuberkulinhauttest oder IFN-y-Sekretion aus T-Lymphozyten) definiert wurde [8]. Auch epidemiologische Betrachtungen sprechen gegen eine signifikante Rolle der B-Lymphozyten. Zwar erkranken Säuglinge am häufigsten an TB, wenn die Antikörperkonzentration in der Muttermilch ihren Nadir erreicht, aber bei Patienten mit Immunglobulin-Mangelsyndromen ist kein gehäuftes Auftreten der TB beschrieben. Beim Common Variable Immunodeficieny Syndrom, das unter anderem über einen Antikörpermangel definiert ist oder unter der Behandlung mit dem Anti-CD52-Antikörper Rituximab, der B-Zellen eliminiert, sind nur vereinzelte TB-Fälle publiziert [70].

Zahlreiche Studien belegen eine große Variabilität der TBspezifischen Antikörper im Bezug auf Antikörperklassen, Antigene, Serumspiegel und Grad der protektiven Wirkung. Eine tabellarische Übersicht der wissenschaftlichen Arbeiten der letzten 7 Jahre wurde kürzlich von Scriba et al. veröffentlicht [8]. Die theoretischen Überlegungen zu antikörpervermittelten Effekten schließen die mykobakterielle Opsonierung, Komplementaktivierung, FcR-vermittelte Phagozytose und die Antigenpräsentation durch B-Zellen in regionalen Lymphknoten ein, jedoch ist die Evidenz für das humane Immunsystem gering [71]. Opsonierte Mykobakterien werden von $M \varphi$ besser phagozytiert. Antikörper gegen Zellwandbestandteile wie Lipoarabinomannan und A-Crystallin können proinflammatorische Prozesse in $M \varphi$ verstärken, die Ausschüttung von IL-6, IFN- $\gamma-S e k r e-$ tion und die Ansäuerung des Phagosoms steigern und somit Mechanismen der angeborenen Immunität unterstützen.

\section{Impfung}

Es liegt nahe, die Fülle an immunologischen Kenntnissen aus Patienten und Tiermodellen zu nutzen, um die Krankheitsentstehung und ihren Verlauf durch eine präventive bzw. therapeutische Impfung zu beeinflussen.

Der weltweit eingesetzte Impfstoff $M$. bovis-BCG ist ein attenuierter Lebendimpfstoff, der Neugeborene vor einer tuberkulösen Meningitis und einer disseminierten Erkrankung, nicht aber vor einer pulmonalen TB schützt $[72,73]$. Dagegen ist bei Erwachsenen und Jugendlichen die $M$. bovis-BCG-Impfung nicht wirksam. Derzeit werden daher Impfstoffe entwickelt, (i) die vor einer Erstinfektion schützen sollen (Priming-Impfstoffe), (ii) die Patienten mit einer latenten Infektion oder nach einer M. bovis-BCG-Impfung verabreicht werden sollen (BoosterImpfstoffe), und (iii) die zusammen mit einer Antibiotikabehandlung verabreicht werden und vor Rezidiven schützen sollen (therapeutische Impfstoffe). Diese Unterscheidung ist wichtig, weil Priming-Impfstoffe Immunantworten gegen Antigene induzieren, die bei der bakteriellen Replikation eine Rolle spielen, während Booster- und therapeutische Impfstoffe Immunantworten gegen Antigene induzieren, die in der latenten Phase exprimiert sind. Multi-Stage-Impfstoffe sollen Immunantworten gegen Antigene induzieren, die sowohl in der akuten als auch der latenten Phase exprimiert werden.

Die Impfstoffentwicklung ist bis heute schwierig, da es kein Tiermodell gibt, das alle wichtigen Aspekte der humanen TB zuverlässig abbildet. Auch ist bis heute kein immunologisches Korrelat der Protektion im Menschen bekannt. Somit kann die protektive Wirkung eines neuen Impfstoffs nur in aufwendigen klinischen Studien untersucht werden. Zur Vereinfachung klinischer Studien können diese auf Individuen aus Hochrisikogruppen wie z.B. Bergleute, die sich vermehrt mit Mtb infizieren, oder Patienten mit einer ausgeheilten TB, die bis zu ca. 10\% ein zweites Mal erkranken, konzentriert werden. Neuere Studien legen nahe, dass es über spezifische Gensignaturen gelingen kann, die TB zu klassifizieren [74]. Sobald auf der Basis solcher Daten valide Biomarker entwickelt werden können, ergeben sich neue Optionen für klinische Impfstoffstudien.

Derzeit befinden sich mehr als ein Dutzend verschiedener Impfstoffkandidaten in der klinischen Entwicklung [75]. Lebend-Impfstoffe sind ursprünglich als Ersatz von $M$. bovis-BCG entwickelt worden, um Neugeborene zu impfen. Ein rekombinanter M. bovis-BCG-Impfstoff (VPM1002) hat in präklinischen Studien eine verbesserte Sicherheit und Wirksamkeit gezeigt. Nach erfolgreichen klinischen Studien der Phasen I und II wird derzeit eine Studie an HIV-infizierten Neugeborenen durchgeführt [76]. In einem nächsten Schritt soll der Impfstoff an Patienten mit einer ausgeheilten TB untersucht werden. Ein anderer Lebendimpfstoff, der auf einer doppelten Deletionsmutante von Mtb basiert (MTBVAC), ist kürzlich erfolgreich in einer Phase-I-Studie untersucht worden [77].

Inaktivierte Erreger stellen einen klassischen Ansatz dar, um Impfstoffe herzustellen. Tatsächlich zeigten bereits vor 70 Jahren erste Impfstudien mit inaktivierten Mykobakterien einen protektiven Effekt. In neueren Studien wurde durch Inaktivierung nicht tuberkulöser Mykobakterien ein Impfstoff herge- 
stellt, der bei HIV-Infizierten in Tansania als Booster-Impfung Schutz vermittelte [78]. Bis heute ist dieser Impfstoff neben $M$. bovis-BCG der einzige, der gegen TB schützt. Zurzeit wird dieser Impfstoff auch als Booster-Impfstoff nach M. bovis-BCG-Impfung untersucht. Ein durch Hitze-Inaktivierung von Mycobacterium vaccae hergestellter Impfstoff wird derzeit in China als therapeutischer Impfstoff erprobt [79].

Untereinheiten-Impfstoffe bestehen aus einem oder mehreren Antigenen, die auf der Basis von präklinischen Tests als protektiv angesehen werden. Solche Impfstoffe werden entweder als rekombinante Proteine zusammen mit Adjuvantien oder als vektorbasierte Impfstoffe hergestellt. Als Vektoren werden Adenovirus-, Vakziniavirus- und Influenzavirus-basierte Systeme eingesetzt, die typischerweise als Boost-Impfstoffe nach M. bovis-BCG-Impfung, zum Teil aber auch in Prime-Boost-Ansätzen untersucht werden. Ein Vektor-basierter Impfstoffkandidat, der auf dem modifizierten Vakziniavirus Ankara (MVA) basiert und das Antigen 85A exprimiert (MVA85), hat in einer klinischen Phase-Ilb-Studie allerdings keine Protektion gezeigt [35].

Solange nicht bekannt ist, welche Antigene von Mtb im Menschen eine protektive Immunantwort auslösen, stellen Lebendimpfstoffe und inaktivierte Impfstoffe eine gute Option für die Impfstoffentwicklung dar. In solchen, auf ganzen bakteriellen Zellen basierenden Impfstoffen sind wahrscheinlich alle bakteriellen Antigene vorhanden. Allerdings kann es sein, dass die protektiven Antigene nur in geringen Konzentrationen vorliegen und dass daher kein guter Immunschutz aufgebaut werden kann. Die Etablierung von Hochdurchsatzmethoden vereinfacht die Suche nach Biomarkern, die möglicherweise Aus-

\section{SCHLUSSFOLGERUNGEN}

Die immunologischen Prozesse, die einer inhalativen Exposition mit Mtb folgen, sind komplex und nur unvollständig verstanden, zumal sie großenteils in der experimentell unzugänglichen Lunge ablaufen. Die systemischen Prozesse spiegeln nur einen kleinen Teil der humanen Immunantwort wider und sind daher in ihrer prognostischen Aussagekraft eingeschränkt. Adaptive und angeborene Mechanismen tragen in vielfältiger Weise zur Eradikation der Erreger bei, sodass die meisten TB-Kontaktpersonen Mtb beseitigen oder kontrollieren können, ohne dass ein immunologischer Nachweis gelingt. Da die derzeit verfügbaren Tiermodelle die humane Immunität nur unvollständig abbilden, sind translationale Forschungsprojekte erforderlich, die auf die Immunologie in der menschlichen Lunge fokussieren. Die derzeitige intensive wissenschaftliche Auseinandersetzung mit dem Mikrobiom des Menschen könnte hierfür förderlich sein. Ein besseres Verständnis dieser Mechanismen könnte zur Entwicklung von neuen adjuvanten Therapien oder Impfstoffen führen, die eine personalisierte Behandlung von Patienten ermöglichen würde. sagen über protektive Antigene zulassen [80]. Es liegen zwar noch keine validierten Biomarker vor, die in der klinischen Praxis Anwendung finden, jedoch konnte in einer Studie bereits mittels einer RNA-Signatur aus einer Blutprobe die Entstehung einer Tuberkulose bei Jugendlichen in Südafrika vorhergesagt werden [81]. Sobald validierte Biomarker vorliegen, ist es wahrscheinlich, dass die Impfstoffentwicklung wichtige neue Impulse bekommt.

\section{Interessenkonflikt}

Die Autoren geben an, dass kein Interessenkonflikt besteht.

\section{Literatur}

[1] Kaufmann SHE. EFIS lecture. Immune response to tuberculosis: How to control the most successful pathogen on earth. Immunol Lett 2016; 175: $50-57$

[2] Liu CH, Liu H, Ge B. Innate immunity in tuberculosis: host defense vs pathogen evasion. Cell Mol Immunol 2017. doi:10.1038/cmi.2017.88

[3] Fennelly KP, Jones-López EC. Quantity and quality of inhaled dose predicts immunopathology in tuberculosis. Front Immunol 2015; 6: 313

[4] Perdomo C, Zedler U, Kühl AA et al. Mucosal BCG vaccination induces protective lung-resident memory $t$ cell populations against Tuberculosis. mBio 2016; 7: e01686-16

[5] Verrall A], Netea MG, Alisjahbana B et al. Early clearance of Mycobacterium tuberculosis: a new frontier in prevention. Immunology 2014; 141: $506-513$

[6] Thoma-Uszynski S, Stenger S, Takeuchi O et al. Induction of direct antimicrobial activity through mammalian toll-like receptors. Science 2001; $291: 1544-1547$

[7] van Tong H, Velavan TP, Thye $\mathrm{T}$ et al. Human genetic factors in tuberculosis: an update. Trop Med Int Health TM IH 2017; 22: 1063 - 1071

[8] Scriba T], Coussens AK, Fletcher HA. Human Immunology of Tuberculosis. Microbiol Spectr 2017; 5: 1-24. doi:10.1128/microbiolspec. TBTB2-0016-2016

[9] Herzmann C, Lange C, Schaberg T et al. Immunological evidence of incipient pulmonary tuberculosis. J Infect Dis 2012; 206: 1630-1631 author reply 1631-1632

[10] Welin A, Raffetseder J, Eklund D et al. Importance of phagosomal functionality for growth restriction of Mycobacterium tuberculosis in primary human macrophages. J Innate Immun 2011; 3: 508 - 518

[11] Sow FB, Florence WC, Satoskar AR et al. Expression and localization of hepcidin in macrophages: a role in host defense against tuberculosis. J Leukoc Biol 2007; 82: 934-945

[12] Martineau AR, Wilkinson KA, Newton SM et al. IFN-gamma- and TNFindependent vitamin D-inducible human suppression of mycobacteria: the role of cathelicidin LL-37. J Immunol Baltim Md 1950 2007; 178: $7190-7198$

[13] Gutierrez MG, Master SS, Singh SB et al. Autophagy is a defense mechanism inhibiting BCG and Mycobacterium tuberculosis survival in infected macrophages. Cell 2004; 119: $753-766$

[14] Chávez-Galán L, Vesin D, Martinvalet D et al. Low Dose BCG Infection as a Model for Macrophage Activation Maintaining Cell Viability. J Immunol Res 2016; 2016: 4048235 
[15] Maeda N, Nigou J, Herrmann J-L et al. The cell surface receptor DCSIGN discriminates between Mycobacterium species through selective recognition of the mannose caps on lipoarabinomannan. J Biol Chem 2003; 278: 5513-5516

[16] Torrelles JB, Azad AK, Schlesinger LS. Fine discrimination in the recognition of individual species of phosphatidyl-myo-inositol mannosides from Mycobacterium tuberculosis by C-type lectin pattern recognition receptors. J Immunol Baltim Md 1950 2006; 177: 1805 - 1816

[17] Behar SM, Carpenter SM, Booty MG et al. Orchestration of pulmonary T cell immunity during Mycobacterium tuberculosis infection: immunity interruptus. Semin Immunol 2014; 26: 559- 577

[18] Lindestam ArlehamnCS, Gerasimova A, Mele F et al. Memory T Cells in Latent Mycobacterium tuberculosis Infection Are Directed against Three Antigenic Islands and Largely Contained in a CXCR3+CCR6+ Th1 Subset. PLoS Pathog 2013; 9: e1003130

[19] Stewart GR, Robertson BD, Young DB. Tuberculosis: a problem with persistence. Nat Rev Microbiol 2003; 1: 97-105

[20] Guirado E, Mbawuike U, Keiser TL et al. Characterization of host and microbial determinants in individuals with latent tuberculosis infection using a human granuloma model. mBio 2015; 6: e02537 - 02514

[21] Gideon HP, Phuah J, Myers AJ et al. Variability in tuberculosis granuloma T cell responses exists, but a balance of pro- and anti-inflammatory cytokines is associated with sterilization. PLoS Pathog 2015; 11: e1004603

[22] Martin C], Cadena AM, Leung VW et al. Digitally carcoding mycobacterium tuberculosis reveals in vivo infection dynamics in the macaque model of tuberculosis. mBio 2017. doi:10.1128/ mBio.00312-17

[23] Malherbe ST, Shenai S, Ronacher K et al. Persisting positron emission tomography lesion activity and Mycobacterium tuberculosis mRNA after tuberculosis cure. Nat Med 2016; 22: 1094-1100

[24] Volkman HE, Pozos TC, Zheng J et al. Tuberculous granuloma induction via interaction of a bacterial secreted protein with host epithelium. Science 2010; 327: $466-469$

[25] Cronan MR, Beerman RW, Rosenberg AF et al. Macrophage epithelial reprogramming underlies mycobacterial granuloma formation and promotes infection. Immunity 2016; 45: 861 - 876

[26] Cyktor JC, Carruthers B, Kominsky RA et al. IL-10 inhibits mature fibrotic granuloma formation during Mycobacterium tuberculosis infection. J Immunol Baltim Md 1950 2013; 190: 2778-2790

[27] Zellweger J-P, Sotgiu G, Block M et al. Risk assessment of tuberculosis in contacts by IFN- $\mathrm{y}$ release assays. A Tuberculosis Network European Trials Group Study. Am J Respir Crit Care Med 2015; 191: 1176 - 1184

[28] Sester M, Sotgiu G, Lange $C$ et al. Interferon-y release assays for the diagnosis of active tuberculosis: a systematic review and meta-analysis. Eur Respir J 2011; 37: 100 - 111

[29] Jafari C, Thijsen S, Sotgiu G et al. Bronchoalveolar lavage enzymelinked immunospot for a rapid diagnosis of tuberculosis: a Tuberculosis Network European Trialsgroup study. Am J Respir Crit Care Med 2009; 180: $666-673$

[30] Filipe-Santos O, Bustamante J, Chapgier A et al. Inborn errors of IL-12/ 23- and IFN-gamma-mediated immunity: molecular, cellular, and clinical features. Semin Immunol 2006; 18: 347 - 361

[31] Lee W-I, Huang J-L, Yeh K-W et al. Immune defects in active mycobacterial diseases in patients with primary immunodeficiency diseases (PIDs). J Formos Med Assoc Taiwan Yi Zhi 2011; 110: 750 - 758

[32] Green AM, Difazio R, Flynn JL. IFN-y from CD4 T cells is essential for host survival and enhances CD8 T cell function during Mycobacterium tuberculosis infection. J Immunol Baltim Md 1950 2013; 190: 270 277

[33] Kagina BMN, Abel B, Scriba T] et al. Specific T cell frequency and cytokine expression profile do not correlate with protection against tuberculosis after bacillus Calmette-Guérin vaccination of newborns. Am J Respir Crit Care Med 2010; 182: 1073-1079

[34] Tsao TCY, Huang CC, Chiou WK et al. Levels of interferon-gamma and interleukin-2 receptor-alpha for bronchoalveolar lavage fluid and serum were correlated with clinical grade and treatment of pulmonary tuberculosis. Int J Tuberc Lung Dis Off J Int Union Tuberc Lung Dis 2002; 6: $720-727$

[35] Tameris MD, Hatherill M, Landry BS et al. Safety and efficacy of MVA85A, a new tuberculosis vaccine, in infants previously vaccinated with BCG: a randomised, placebo-controlled phase $2 \mathrm{~b}$ trial. Lancet Lond Engl 2013; 381: $1021-1028$

[36] Herzmann C, Ernst M, Ehlers $S$ et al. Increased frequencies of pulmonary Treg cells in latent M. tuberculosis infection. Eur Respir J 2012; 40: $1450-1457$

[37] Semple PL, Binder AB, Davids M et al. Regulatory T cells attenuate mycobacterial stasis in alveolar and blood-derived macrophages from patients with tuberculosis. Am J Respir Crit Care Med 2013; 187: $1249-1258$

[38] Henao-Tamayo MI, Obregón-Henao A, Arnett K et al. Effect of bacillus Calmette-Guérin vaccination on CD4+Foxp3+T cells during acquired immune response to Mycobacterium tuberculosis infection. J Leukoc Biol 2016; 99: 605-617

[39] Srivastava S, Ernst JD. Cutting edge: Direct recognition of infected cells by CD4 T cells is required for control of intracellular Mycobacterium tuberculosis in vivo. J Immunol Baltim Md 1950 2013; 191: $1016-1020$

[40] Kalsdorf B, Scriba T], Wood K et al. HIV-1 infection impairs the bronchoalveolar T-cell response to mycobacteria. Am J Respir Crit Care Med 2009; 180: $1262-1270$

[41] Jambo KC, Sepako E, Fullerton DG et al. Bronchoalveolar CD4+ T cell responses to respiratory antigens are impaired in HIV-infected adults. Thorax 2011; 66: 375 - 382

[42] Sakai S, Kauffman KD, Schenkel JM et al. Cutting edge: control of Mycobacterium tuberculosis infection by a subset of lung parenchymahoming CD4 T cells. J Immunol Baltim Md 1950 2014; 192: 2965 2969

[43] Sakai S, Kauffman KD, Sallin MA et al. CD4 T Cell-Derived IFN-y Plays a Minimal Role in Control of Pulmonary Mycobacterium tuberculosis Infection and Must Be Actively Repressed by PD-1 to Prevent Lethal Disease. PLoS Pathog 2016; 12: e1005667

[44] Sousa AO, Mazzaccaro RJ, Russell RG et al. Relative contributions of distinct MHC class I-dependent cell populations in protection to tuberculosis infection in mice. Proc Natl Acad Sci U S A 2000; 97: 4204 4208

[45] Silva BD de S, Trentini MM, da Costa AC et al. Different phenotypes of $\mathrm{CD} 8+\mathrm{T}$ cells associated with bacterial load in active tuberculosis. Immunol Lett 2014; 160: $23-32$

[46] van Pinxteren LA, Cassidy JP, Smedegaard BH et al. Control of latent Mycobacterium tuberculosis infection is dependent on CD8 T cells. Eur J Immunol 2000; 30: 3689-3698

[47] Ghazalsofala R, Rezaee SA, Rafatpanah $\mathrm{H}$ et al. Evaluation of CD4+ CD25+ FoxP3+ Regulatory T cells and FoxP3 and CTLA-4 gene Expression in Patients with Newly Diagnosed Tuberculosis in Northeast of Iran. Jundishapur J Microbiol 2015; 8: e17726

[48] Li N, Xie W-P, Kong H et al. Enrichment of regulatory T-cells in blood of patients with multidrug-resistant tuberculosis. Int J Tuberc Lung Dis Off J Int Union Tuberc Lung Dis 2015; 19: 1230-1238

[49] Wang C, jiang T, Wei L et al. Association of CTLA4 gene polymorphisms with susceptibility and pathology correlation to pulmonary tuberculosis in Southern Han Chinese. Int J Biol Sci 2012; 8: 945- 952

[50] Kirman ], McCoy K, Hook S et al. CTLA-4 blockade enhances the immune response induced by mycobacterial infection but does not lead to increased protection. Infect Immun 1999; 67: 3786-3792 
[51] Amulic B, Cazalet C, Hayes GL et al. Neutrophil function: from mechanisms to disease. Annu Rev Immunol 2012; 30: 459-489

[52] Eum SY, Kong JH, Hong MS et al. Neutrophils are the predominant infected phagocytic cells in the airways of patients with active pulmonary TB. Chest 2009; 137: $122-128$

[53] Gopal R, Monin L, Torres D et al. S100A8/A9 proteins mediate neutrophilic inflammation and lung pathology during tuberculosis. Am J Respir Crit Care Med 2013; 188: 1137-1146

[54] Berrocal-Almanza LC, Goyal S, Hussain A et al. S100A12 is up-regulated in pulmonary tuberculosis and predicts the extent of alveolar infiltration on chest radiography: an observational study. Sci Rep 2016; 6: 31798

[55] Berry MPR, Graham CM, McNab FW et al. An interferon-inducible neutrophil-driven blood transcriptional signature in human tuberculosis. Nature 2010; 466: 973-977

[56] Dallenga T, Schaible UE. Neutrophils in tuberculosis - first line of defence or booster of disease and targets for host directed therapy? Pathog Dis 2016; doi: doi:10.1093/femspd/ftw012

[57] Dallenga T, Repnik U, Corleis B et al. M. tuberculosis-Induced Necrosis of Infected Neutrophils Promotes Bacterial Growth Following Phagocytosis by Macrophages. Cell Host Microbe 2017; 22: 519-530.e3

[58] Corleis B, Korbel D, Wilson R et al. Escape of Mycobacterium tuberculosis from oxidative killing by neutrophils. Cell Microbiol 2012; 14 : $1109-1121$

[59] Manca C, Paul S, Barry CE et al. Mycobacterium tuberculosis catalase and peroxidase activities and resistance to oxidative killing in human monocytes in vitro. Infect Immun 1999; 67: 74-79

[60] Millman AC, Salman M, Dayaram YK et al. Natural killer cells, glutathione, cytokines, and innate immunity against Mycobacterium tuberculosis. J Interferon Cytokine Res Off J Int Soc Interferon Cytokine Res 2008; 28: 153-165

[61] Guerra C, Johal K, Morris D et al. Control of Mycobacterium tuberculosis growth by activated natural killer cells. Clin Exp Immunol 2012; 168: $142-152$

[62] Allen M, Bailey C, Cahatol I et al. Mechanisms of Control of Mycobacterium tuberculosis by NK Cells: Role of Glutathione. Front Immunol 2015; 6: 508

[63] Schierloh P, Alemán M, Yokobori N et al. NK cell activity in tuberculosis is associated with impaired CD11a and ICAM-1 expression: a regulatory role of monocytes in NK activation. Immunology 2005; 116 : $541-552$

[64] Junqueira-Kipnis AP, Kipnis A, Jamieson A et al. NK cells respond to pulmonary infection with Mycobacterium tuberculosis, but play a minimal role in protection. J Immunol Baltim Md 1950 2003; 171: 6039-6045

[65] Jiang J, Yang B, An H et al. Mucosal-associated invariant T cells from patients with tuberculosis exhibit impaired immune response. J Infect 2016; 72: 338-352

[66] Sakala IG, Kjer-Nielsen L, Eickhoff CS et al. Functional Heterogeneity and Antimycobacterial Effects of Mouse Mucosal-Associated Invariant
T Cells Specific for Riboflavin Metabolites. J Immunol Baltim Md 1950 2015; 195: 587-601

[67] Boom WH. Gammadelta T cells and Mycobacterium tuberculosis. Microbes Infect 1999; 1: 187-195

[68] Meraviglia S, El DakerS, Dieli F et al. үઈ T cells cross-link innate and adaptive immunity in Mycobacterium tuberculosis infection. Clin Dev Immunol 2011; 2011: 587315. doi:10.1155/2011/587315

[69] Achkar JM, Chan J, Casadevall A. B cells and antibodies in the defense against Mycobacterium tuberculosis infection. Immunol Rev 2015; 264: $167-181$

[70] Alkadi A, Alduaiji N, Alrehaily A. Risk of tuberculosis reactivation with rituximab therapy. Int J Health Sci 2017; 11: $41-44$

[71] Achkar JM, Chan J, Casadevall A. B cells and antibodies in the defense against Mycobacterium tuberculosis infection. Immunol Rev 2015; 264: $167-181$

[72] Colditz GA, Berkey CS, Mosteller F et al. The efficacy of bacillus Calmette-Guérin vaccination of newborns and infants in the prevention of tuberculosis: meta-analyses of the published literature. Pediatrics 1995; 96: 29-35

[73] Colditz GA, Brewer TF, Berkey CS et al. Efficacy of BCG vaccine in the prevention of tuberculosis. Meta-analysis of the published literature. JAMA 1994; 271: 698-702

[74] Maertzdorf J, McEwen G, Weiner J et al. Concise gene signature for point-of-care classification of tuberculosis. EMBO Mol Med 2016; 8: $86-95$

[75] Kaufmann SHE, Weiner J, von Reyn CF. Novel approaches to tuberculosis vaccine development. Int J Infect Dis IJID Off Publ Int Soc Infect Dis 2017; 56: $263-267$

[76] Loxton AG, Knaul JK, Grode L et al. Safety and Immunogenicity of the Recombinant Mycobacterium bovis BCG Vaccine VPM1002 in HIVUnexposed Newborn Infants in South Africa. Clin Vaccine Immunol CVI 2017. doi:10.1128/CVI.00439-16

[77] Spertini F, Audran R, Chakour R et al. Safety of human immunisation with a live-attenuated Mycobacterium tuberculosis vaccine: a randomised, double-blind, controlled phase I trial. Lancet Respir Med 2015; 3: $953-962$

[78] von Reyn CF, Mtei L, Arbeit RD et al. Prevention of tuberculosis in Bacille Calmette-Guérin-primed, HIV-infected adults boosted with an inactivated whole-cell mycobacterial vaccine. AIDS 2010; 24 : 675 685

[79] Weng H, Huang J-Y, Meng X-Y et al. Adjunctive therapy of Mycobacterium vaccae vaccine in the treatment of multidrug-resistant tuberculosis: A systematic review and meta-analysis. Biomed Rep 2016; 4: 595-600

[80] Weiner ], Kaufmann SHE. High-throughput and computational approaches for diagnostic and prognostic host tuberculosis biomarkers. Int J Infect Dis IJID Off Publ Int Soc Infect Dis 2017; 56: 258-262

[81] Zak DE, Penn-Nicholson A, Scriba T] et al. A blood RNA signature for tuberculosis disease risk: a prospective cohort study. Lancet 2016; 387: $2312-2322$ 